(c) 2020 Universidad Nacional Autónoma de México, Facultad de Estudios Superiores Zaragoza.

Este es un artículo Open Access bajo la licencia CC BY-NC-ND (http://creativecommons.org/licenses/by-nc-nd/4.0/).

TIP Revista Especializada en Ciencias Químico-Biológicas, 23: 1-7, 2020.

https://doi.org/10.22201/fesz.23958723e.2020.0.272

\title{
Evidencias de transmisión intergeneracional de la obesidad y sus comorbilidades
}

\author{
Carlos Alfonso Larqué Velázquez y Jose Rene Escalona Mugica* \\ Departamento de Embriología y Genética, Facultad de Medicina, Universidad Nacional \\ Autónoma de México. Avenida Universidad \# 3000, Col. Ciudad Universitaria, Alcaldía \\ de Coyoacán 04510, Ciudad de México, México. E-mail: *escalonarj@facmed.unam.mx
}

\begin{abstract}
RESUMEN
Actualmente, a nivel mundial se está atravesando por una transición epidemiológica en la que las enfermedades infecciosas han dejado de ser las principales causas de muerte, ahora las enfermedades crónico-degenerativas son cada vez más prevalentes. El aumento global del sobrepeso y la obesidad tiene un papel primordial en esta transición, siendo factores de riesgo para desarrollar desórdenes metabólicos, enfermedades cardiovasculares y algunos tipos de cáncer. Por lo tanto, resulta una prioridad el estudio de todos los factores asociados al desarrollo del sobrepeso y la obesidad. El objetivo de esta nota científica, es señalar que la obesidad y sus comorbilidades tienen efectos sobre la programación metabólica durante la gestación, por lo que pueden aumentar el riesgo en la descendencia (más allá de una generación) de desarrollarlas. La hipótesis de que las enfermedades crónico-degenerativas (obesidad, hipertensión, diabetes mellitus tipo 2 o enfermedades cardiovasculares), que generalmente se presentan en la edad adulta se originen durante el desarrollo del individuo parece poco probable. Sin embargo, existe evidencia tanto epidemiológica como experimental, que demuestra que el estado nutricional y metabólico de ambos padres previo a la concepción y de la madre durante la gestación, pueden repercutir en la salud de su progenie durante la vida adulta.
\end{abstract}

Palabras clave: obesidad, intergeneracionalidad, herencia, metabolismo, desarrollo, programación metabólica.

\section{Evidence for the intergenerational transmission of obesity and its comorbidities}

\begin{abstract}
Currently, the world is experiencing an epidemiological transition; where infectious diseases are not among the main causes of death; while the prevalence of chronic and degenerative diseases is increasing. The global rise in the incidence of overweight and obese individuals is one of the main drivers of this change; both are risk factors to develop metabolic anomalies, cardiovascular diseases (CVDs) and some types of cancer. Therefore, the study of all the factors involved in the development of obesity is a priority. The aim of this short review is to point out that obesity, as well as its comorbidities, can be primed during gestation and can be passed through more than one subsequent generation. At first glance, the notion that chronic and degenerative diseases, which usually manifest at adult age (e.g., obesity, hypertension, type 2 diabetes mellitus, CVDs), could have a developmental origin seems implausible. Nevertheless, there is both epidemiological and experimental evidence that support the idea that, the nutritional and metabolic state of the parents prior to conception, and of the mother during pregnancy, can influence the adult life of their offspring. Keywords: obesity, intergenerational, heredity, metabolism, development, metabolic programming.
\end{abstract}




\section{INTRODUCCIÓN}

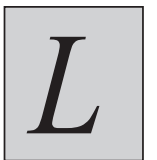

a obesidad es actualmente un serio problema de salud pública, ya que de acuerdo con la Organización Mundial de la Salud (OMS), en los últimos 45 años su incidencia se ha triplicado a nivel mundial. En México, la Encuesta Nacional de Salud y Nutrición 2018, reportó que el $76.8 \%$ de la población adulta presenta sobrepeso u obesidad (Secretaría de Salud, Instituto Nacional de Salud Pública e Instituto Nacional de Estadística y Geografía, 2018). Dada la importancia que representan la obesidad y sus comorbilidades, además de la posibilidad de que estas puedan afectar a las futuras generaciones, resultan de gran interés científico. Uno de los indicadores más utilizados para evaluar el sobrepeso y la obesidad es el índice de masa corporal (IMC), que es la relación entre la masa corporal y la talla en metros al cuadrado. De acuerdo con la OMS, el IMC permite categorizar a las personas en un peso normal (18.5-25 $\left.\mathrm{kg} / \mathrm{m}^{2}\right)$, sobrepeso $\left(>25-29.9 \mathrm{~kg} / \mathrm{m}^{2}\right)$ y obesidad $\left(\geq 30 \mathrm{~kg} / \mathrm{m}^{2}\right)$ (OMS, 2020).

La programación metabólica implica al ambiente en el que se desarrolla un individuo (en ventanas críticas del desarrollo), el cual ejerce efectos que determinan y establecen características morfofisiológicas. El exponer a un individuo a un ambiente nutricionalmente adverso, ya sea por la falta o exceso de macronutrientes durante su vida intrauterina y postnatal temprana, aumenta la susceptibilidad de que éste desarrolle enfermedades metabólicas en la edad adulta. Desde 1975, se reconoció que los cambios en variables como los niveles plasmáticos de las hormonas, neurotransmisores y metabolitos, durante un periodo crítico del desarrollo, es decir, durante el desarrollo pre- y perinatal de los sistemas neuroendocrinos, son capaces de "programar o preparar" al producto tanto a nivel metabólico como conductual (Dörner, 1975). En 1990, se realizó uno de los primeros estudios en 424 niños pretérmino, analizando los efectos a largo plazo producidos por la dieta del neonato durante las primeras semanas de vida. El estudio comparó los efectos producidos por una fórmula estándar, con aquellos producidos por una fórmula especial, o sea, con mayor contenido proteico y una mayor densidad energética diseñada especialmente para niños pretérmino. Los resultados del estudio mostraron, que la dieta del neonato durante ese corto periodo de tiempo, tenía consecuencias a largo plazo sobre el desarrollo neurológico. Además, se observó que, a los 18 meses de vida, los niños alimentados con la fórmula especial tenían un mejor desarrollo psicomotor, en comparación con aquellos alimentados con la fórmula estándar (Lucas et al., 1990). A partir de entonces, múltiples estudios epidemiológicos han aportado evidencias sobre el fenómeno de programación metabólica y el aumento de la susceptibilidad de padecer obesidad y sus comorbilidades. En las siguientes secciones, se discutirán las evidencias obtenidas de estudios realizados en humanos y modelos animales sobre cómo la obesidad parental puede impactar el desarrollo y la salud de las futuras generaciones.

\section{EVIDENCIAS EPIDEMIOLÓGICAS DE LA PROGRAMACIÓN METABÓLICA Y LA SUSCEPTIBILIDAD A DESARROLLAR OBESIDAD}

Un estudio clave en el área de la programación metabólica, viene del análisis de las consecuencias de la hambruna sufrida en el invierno de 1944, en los Países Bajos durante la segunda guerra mundial. Debido a la ocupación alemana, se presentó una escasez de alimentos durante este periodo. La ingesta calórica de toda la población, incluyendo a las mujeres embarazadas, disminuyó de forma significativa llegando a ser inclusive de 400-600 kilocalorías por día. Posterior a la guerra, esta región atravesó un periodo de relativa prosperidad, que proporcionó una oportunidad única, para evaluar cómo la disponibilidad de nutrientes puede afectar el desarrollo y la salud a lo largo de la vida de la progenie (Schulz, 2010). Por ejemplo, se observó que los hijos adultos de hombres expuestos a la restricción calórica presentaban un IMC mayor al de individuos nacidos de padres que no estuvieron expuestos a tal evento (Veenendal et al., 2013). Adicionalmente, se observó que las hijas de madres expuestas a la hambruna, presentaban alteraciones en el perfil de lípidos (aumento en la concentración de colesterol total, colesterol LDL y triacilgliceroles), en comparación con mujeres nacidas después de ese periodo (Lumey, Stein, Kahn \& Romijn, 2009). A la fecha, se han publicado más de 140 estudios analizando este fenómeno, en los que se han encontrado múltiples asociaciones entre la disponibilidad de alimento en edades tempranas y alteraciones metabólicas en la edad adulta. Dichas alteraciones no están limitadas a los individuos directamente expuestos, sino que, además, pueden tener consecuencias en el desarrollo y la salud de su progenie. Este fenómeno no está limitado a la malnutrición y la restricción calórica, sino que, además, ocurre con la obesidad.

En este sentido, se ha observado que las mujeres con obesidad y/o con diabetes mellitus tipo 2 (DM2) presentan un riesgo mayor de gestar niños con diabetes mellitus tipo 1 (DM1). Asimismo, el aumento del IMC materno se correlaciona con una manifestación más temprana de la DM1 (Lindell, Carlsson, Josefsson \& Samuelsson, 2018). En un estudio realizado en los Países Bajos, se evaluó si el estado metabólico de las madres al momento de la concepción, se correlacionaba con las características antropométricas y el riesgo cardiovascular de sus hijos a los 6 años de edad. En este estudio, se encontró que las mujeres con obesidad, presentaban una mayor probabilidad de tener hijos que desarrollarán obesidad a edad temprana. Además, se encontró que los hijos de madres con obesidad pregestacional mostraban un riesgo mayor de presentar un porcentaje elevado de grasa abdominal, así como aumento en la presión arterial sistólica, en la concentración de insulina y en el riesgo cardio-metabólico, en comparación con 
los hijos de mujeres con peso normal (Gaillard et al., 2014). Por otra parte, también se ha obtenido información sobre el aumento de susceptibilidad de la descendencia a desarrollar hígado graso no alcohólico (HGNA), como consecuencia de la obesidad materna. Se ha demostrado que los niños nacidos de madres con obesidad, presentan un aumento en la cantidad de grasa hepática, en comparación con los niños de madres con un IMC normal; cabe señalar que este, se presenta aún sin que exista un incremento en la cantidad de grasa subcutánea (Brumbaugh et al., 2013). Adicionalmente, dentro de las complicaciones observadas en madres obesas se encuentran el que se acreciente la probabilidad de nacimientos pretérmino y la presencia de un puntaje bajo en la prueba de APGAR (escala de evaluación que se obtiene en los primeros minutos de vida, que valora el estado general del recién nacido, a través de la examinación del tono muscular, respiración, frecuencia cardiaca, reflejos y color de piel) (Sebire et al., 2001). Adicionalmente, los hijos de madres con obesidad, presentan una mayor incidencia de problemas respiratorios en los primeros meses de vida (Håberg, Stigum, London, Nystad \& Nafstad, 2009). Otro de los sistemas que resultan afectados por la exposición intrauterina a un ambiente obesogénico, es el sistema reproductivo. En este sentido, se han realizado estudios en los que se ha observado, que las mujeres gestadas por madres obesas, presentan un riesgo mayor de desarrollar síndrome de ovario poliquístico en la edad adulta, en comparación con mujeres gestadas por madres con un peso normal (Cresswell et al., 1997).

Cabe señalar, que la mayoría de los estudios se ha enfocado en el efecto de la obesidad materna sobre la progenie. Probablemente, debido a que generalmente se considera que la contribución paterna al desarrollo del producto queda limitada al espermatozoide, mientras que el aporte materno además del ovocito, incluye al microambiente necesario para mantener el desarrollo del producto durante la gestación. Sin embargo, cada vez existe más evidencia sobre los efectos negativos de la obesidad (y otros elementos del estilo de vida) paterna sobre el desarrollo de la progenie. Un estudio multigeneracional en Suecia, encontró que la disponibilidad de comida en la etapa prepuberal de los abuelos paternos, se relacionaba con la causa de muerte (DM2 y enfermedades cardiovasculares) de sus nietos (Kaati, Bygren \& Edvinsson, 2002). Adicionalmente, un estudio que incluyó a parejas no obesas, encontró que el IMC paterno se correlacionaba con el IMC de los hijos, a partir de las 12 semanas de vida posnatal. Además, se observó que este efecto era independiente y aditivo al efecto del IMC materno (Knight et al., 2007). Asimismo, otro estudio mostró que la presencia de DM2 y/o de síndrome metabólico (la manifestación de al menos tres de los siguientes: obesidad central, triacilgliceroles $\geq$ $150 \mathrm{mg} / \mathrm{dL}$, colesterol HDL $\leq 40 \mathrm{mg} / \mathrm{dl}$, tensión arterial $\geq$ $130 / 85 \mathrm{mmHg}$ y glucosa en ayuno $\geq 100 \mathrm{y}<126 \mathrm{mg} / \mathrm{dl}$ ) en el padre, aumentaba la probabilidad de que su descendencia presentara un IMC elevado (Linares Segovia, Gutiérrez Tinoco, Izquierdo Arrizon, Guízar Mendoza \& Amador Licona, 2012).

\section{EVIDENCIAS EN MODELOS ANIMALES}

Una de las limitantes que pueden presentar los estudios epidemiológicos en humanos debido a su diseño, es la inclusión de factores de confusión por variables no controladas relacionadas con el estilo de vida de las personas o con el medio ambiente, que impiden la obtención de conclusiones definitivas. Asimismo, este tipo de estudios permiten la identificación de correlaciones, aunque estas no necesariamente impliquen causalidad. Es aquí, donde los estudios en modelos animales proveen una potente herramienta, para eliminar factores de confusión y controlar la mayor cantidad de posibles variables.

El uso de cepas endogámicas de roedores, ha permitido reducir la interferencia causada por la variabilidad genética, misma que es ineludible en humanos. Además, las condiciones controladas en un bioterio permiten la evaluación de variables de forma independiente. Los modelos animales también permiten la evaluación no sólo de relaciones causales, sino también de los mecanismos epigenéticos, moleculares y/o bioquímicos involucrados en la transmisión de estos rasgos. Finalmente, la mayoría de los modelos animales tiene la ventaja adicional de tener tiempos intergeneracionales más cortos que los humanos, lo que facilita el estudio de fenómenos multigeneracionales.

Los roedores constituyen algunos de los modelos animales más utilizados para el estudio de la programación metabólica, debido a que múltiples procesos metabólicos, como la homeostasis de la glucosa, el metabolismo de lípidos y el control de la reproducción entre otros, son similares a los de los humanos. Asimismo, la exposición de múltiples cepas de roedores a dietas hipercalóricas, genera obesidad. Diversos estudios en ratones con obesidad inducida por dieta (OID) alta en grasas, han mostrado que las crías de ratones hembra obesas, presentan un mayor consumo calórico diario (independientemente de la densidad calórica de su dieta), una mayor ganancia de peso y una mayor proporción de tejido adiposo, en comparación con las crías de ratones hembra con un peso normal. Además, se ha observado que la progenie de madres obesas, presenta anomalías metabólicas como hiperleptinemia, hiperinsulinemia e hipertensión (Samuelsson et al., 2008). Un estudio posterior demostró que las crías de madres obesas, no sólo presentaban hiperleptinemia, sino además, desarrollaban resistencia al efecto anorexigénico producido por la administración de leptina exógena (Kirk et al., 2009). Esto sugiere que la obesidad materna puede programar a la progenie a desarrollar una mayor ingesta calórica, mediante una resistencia a la acción de la leptina en el hipotálamo. 
Por otro lado, experimentos realizados en roedores, han brindado evidencia del potencial efecto negativo que tiene la obesidad paterna sobre la descendencia. En este sentido, en un modelo de ratas con OID, se observó que los machos obesos heredan una disfunción pancreática a su progenie femenina ( $\mathrm{Ng}$ et al.,2010). Esta disfunción se caracterizó por la presencia de alteraciones en la secreción de insulina, intolerancia a la glucosa y cambios en la expresión de 642 genes, asociados a la funcionalidad de los islotes pancreáticos. Un estudio posterior, ahondó en la programación metabólica transmitida por vía paterna en ratas. En este, se demostró que la obesidad paterna inducida por dieta, favorece el desarrollo de intolerancia a la glucosa en las descendientes hembras (F1). Además, se observó que este fenotipo persistía hasta una generación más (F2), a pesar de que los animales de las generaciones F1 y F2, fueron alimentados exclusivamente con una dieta normocalórica. Finalmente, en este mismo estudio se encontró que los patrones de metilación del DNA, en los espermatozoides de ratas F0, son similares a los de los espermatozoides de las ratas F2, lo cual sugiere una transmisión epigenética (de Castro Barbosa et al., 2015). Es decir, estos rasgos no se transmiten por cambios en la secuencia de nucleótidos del DNA, sino mediante modificaciones covalentes (metilaciones, acetilaciones, fosforilaciones, etc) sobre las bases nitrogenadas o las histonas, que pueden modificar la expresión génica.

A pesar de que generalmente se asume que la transmisión de la obesidad y sus anomalías asociadas, es epigenética, tanto por vía materna (Godfrey et al., 2017) como por vía paterna (Slyvka, Zhang \& Nowak, 2015), se ha demostrado que los efectos intergeneracionales de la obesidad, no están restringidos al genoma nuclear. Existen estudios que han observado que la descendencia de ratones hembra con obesidad, presenta cambios en la morfología mitocondrial, en la cantidad de DNA mitocondrial, así como en la expresión de los componentes de la cadena respiratoria, tanto en el músculo estriado como en los ovocitos, hasta por dos generaciones subsecuentes (Saben et al., 2016). Finalmente, a pesar de las múltiples ventajas que presentan los modelos murinos, algunos procesos fisiológicos y patológicos en humanos, no tienen un equivalente en ratas o ratones. Por lo tanto, se han desarrollado modelos de estudio de la programación metabólica en primates no humanos $(\mathrm{PNH})$ en especies como el macaco japonés (Macaca fuscata). La importancia de este novedoso modelo animal, radica en la semejanza de los procesos del desarrollo de la placenta, de los islotes pancreáticos y del cerebro con los del humano (Isa, Yamane, Hamai \& Inagaki, 2009). Por otra parte, los macacos manifiestan algunos aspectos psico-sociales que pueden ser estudiados y extrapolados a los humanos (Friedman, 2018). En este modelo de PNH, se ha observado que la administración de una dieta hipercalórica, no sólo aumenta el peso de las hembras gestantes, sino además, induce un aumento en la adiposidad y en el depósito hepático de lípidos compatible con la presencia de HGNA en la progenie (McCurdy et al., 2009). Además, otros estudios han observado, que estas alteraciones se encuentran asociadas a cambios en las marcas epigenéticas de las histonas de células del tejido hepático (Aagaard-Tillery et al., 2008).

Cabe destacar que en el mismo modelo de $\mathrm{PNH}$, se ha observado que la transmisión de las alteraciones metabólicas, no está limitada a mecanismos epigenéticos. Se ha demostrado que una dieta hipercalórica, no sólo cambia el microbioma materno, sino que es capaz de alterar el microbioma de su descendencia (Ma et al., 2014). Estos resultados proveen evidencia de que la dieta materna, puede influir en la salud metabólica de forma intergeneracional, mediante sus efectos, sobre el establecimiento de la microbiota en la descendencia. Actualmente, existe controversia sobre la transferencia vertical in utero del microbioma, sin embargo, diversos trabajos han demostrado que el ambiente intrauterino y la dieta materna, influyen en el establecimiento del microbioma de la progenie (Zhou \& Xiao, 2018; Li, 2018). El trabajo con modelos en ratones, ha permitido observar que la obesidad materna se asocia no sólo a un aumento del riesgo de desarrollar HGNA y obesidad, sino también a la alteración del microbioma de la progenie. Un estudio demostró que los ratones hijos de madres obesas, presentan una disminución en la proporción de bacterias del género Bacteroidetes y Gammaproteobacteria, así como un aumento en la proporción de bacterias del género Firmicutes en comparación con los ratones hijos de madres con peso normal. Además, se observó que los cambios en el microbioma, se acompañaban de un aumento en la permeabilidad intestinal y alteraciones en la funcionalidad de los macrófagos. Por lo tanto, se ha propuesto que estas alteraciones estructurales e inmunológicas, podrían favorecer la colonización intestinal por microorganismos relacionados con la obesidad y el desarrollo de un estado proinflamatorio, en etapas tempranas de la vida posnatal de la progenie (Hernandez, Friedman \& Barbour, 2020; Soderborg et al., 2018). Finalmente, la microbiota materna podría también influir directamente en la programación metabólica de la progenie. Se ha observado, que algunos metabolitos generados por la microbiota intestinal, como los ácidos grasos de cadena corta, pueden atravesar la barrera placentaria hacia la circulación fetal (Gomez de Agüero et al., 2016). Los ácidos grasos de cadena corta son capaces de activar receptores acoplados a proteínas $\mathrm{G}$, así como de modular algunos aspectos del metabolismo, el funcionamiento de la vesícula biliar y la secreción de hormonas entéricas hacia la circulación sistémica (Barathikannan et al., 2019).

\section{Conclusiones}

El sobrepeso y la obesidad, así como sus complicaciones asociadas, representan un serio problema de salud pública. En conjunto, diversos estudios proveen evidencia de que el 
estado metabólico y nutricional de los padres, durante un periodo crítico del desarrollo del individuo, pueden influir en el desarrollo intrauterino a través de distintos mecanismos y aumentar la susceptibilidad del producto, a presentar anomalías metabólicas a lo largo de su vida. Además, los efectos de la obesidad de cualquiera de los progenitores, son heredables más allá de una sola generación (Figura 1).

Por lo tanto, la programación metabólica y la transmisión intergeneracional de la susceptibilidad a la obesidad, son áreas que requieren una mayor atención. La determinación de los mecanismos moleculares involucrados en estos fenómenos, resultará de gran valor en el diseño de intervenciones terapéuticas, así como en la identificación de biomarcadores y factores de riesgo específicos. Finalmente, los diversos descubrimientos en estas áreas, sentarán las bases en el diseño de estrategias de salud pública, encaminadas a la prevención de la obesidad y sus comorbilidades en futuras generaciones.

\section{Agradecimientos}

Agradecemos al Departamento de Embriología y Genética y a la División de Investigación de la Facultad de Medicina, UNAM, por el apoyo técnico y financiamiento.

\section{REFERENCIAS}

Aagaard-Tillery, K. M., Grove, K., Bishop, J., Ke, X., Fu, Q., McKnight, R. \& Lane, R. H. (2008). Developmental origins of disease and determinants of chromatin structure: maternal diet modifies the primate fetal epigenome. Journal of Molecular Endocrinology, 41(2), 91-102. https://doi.org/10.1677/JME-08-0025

Barathikannan, K., Chelliah, R., Rubab, M., Daliri, E. B., Elahi, F., Kim, D. H., Agastian, P., Oh, S. Y. \& Oh, D. H. (2019). Gut Microbiome Modulation Based on Probiotic Application for Anti-Obesity: A Review on Efficacy and Validation. Microorganisms, 7(10), 456. https://doi. org/10.3390/microorganisms 7100456

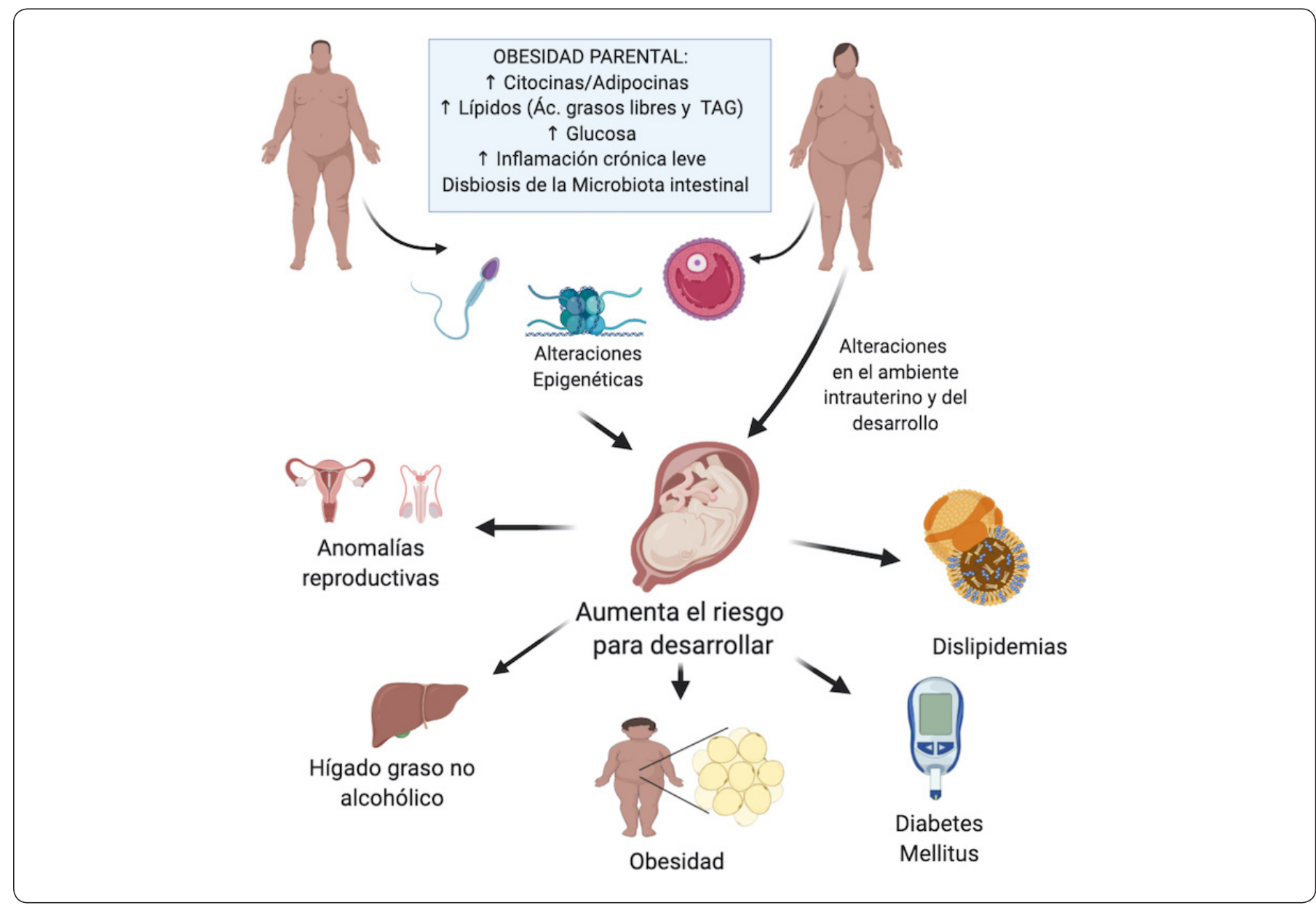

Figura 1. Alteraciones asociadas a la obesidad parental. La obesidad parental, está asociada a múltiples anomalías metabólicas, que pueden alterar marcas epigenéticas en los gametos; así, como alterar las condiciones en las cuales se desarrolla el producto. Estos cambios pueden predisponer a la progenie de padres adultos, a desarrollar diversas alteraciones metabólicas. Imagen de creación propia mediante Biorender.com (2020). 
Brumbaugh, D. E., Tearse, P., Cree-Green, M., Fenton, L. Z., Brown, M., Scherzinger, A., Reynolds, R., Alston, M., Hoffman, C., Pan, Z., Friedman, J. E. \& Barbour, L. A. (2013). Intrahepatic fat is increased in the neonatal offspring of obese women with gestational diabetes. The Journal of pediatrics, 162(5), 930-6.e1. https://doi. org/10.1016/j.jpeds.2012.11.017

Cresswell, J. L., Barker, D. J., Osmond, C., Egger, P., Phillips, D. I. \& Fraser, R. B. (1997). Fetal growth, length of gestation, and polycystic ovaries in adult life. Lancet (London, England), 350(9085), 1131-1135. https://doi. org/10.1016/s0140-6736(97)06062-5

de Castro Barbosa, T., Ingerslev, L. R., Alm, P. S., Versteyhe, S., Massart, J., Rasmussen, M., Donkin, I., Sjögren, R., Mudry, J. M., Vetterli, L., Gupta, S., Krook, A., Zierath, J. R., \& Barrès, R. (2015). High-fat diet reprograms the epigenome of rat spermatozoa and transgenerationally affects metabolism of the offspring. Molecular metabolism, 5(3), 184-197. https://doi.org/10.1016/j. molmet.2015.12.002

Dörner, G. (1975). Perinatal hormone levels and brain organization. In Anatomical neuroendocrinology (pp. 245-252). Karger Publishers.

Friedman, J. E. (2018). Developmental Programming of Obesity and Diabetes in Mouse, Monkey, and Man in 2018: Where Are We Headed? Diabetes, 67(11), 2137 2151. https://doi.org/10.2337/dbi17-0011

Gaillard, R., Steegers, E. A., Duijts, L., Felix, J. F., Hofman, A., Franco, O. H. \& Jaddoe, V. W. (2014). Childhood cardiometabolic outcomes of maternal obesity during pregnancy: the Generation R Study. Hypertension (Dallas, Tex. : 1979), 63(4), 683-691. https://doi.org/10.1161/ HYPERTENSIONAHA.113.02671

Godfrey, K. M., Reynolds, R. M., Prescott, S. L., Nyirenda, M., Jaddoe, V. W., Eriksson, J. G. \& Broekman, B. F. (2017). Influence of maternal obesity on the longterm health of offspring. The lancet. Diabetes \& endocrinology, 5(1), 53-64. https://doi.org/10.1016/ S2213-8587(16)30107-3

Gomez de Agüero, M., Ganal-Vonarburg, S. C., Fuhrer, T., Rupp, S., Uchimura, Y., Li, H., Steinert, A., Heikenwalder, M., Hapfelmeier, S., Sauer, U., McCoy, K. D. \& Macpherson, A. J. (2016). The maternal microbiota drives early postnatal innate immune development. Science (New York, N.Y.), 351(6279), 1296-1302. https:// doi.org/10.1126/science.aad2571

Håberg, S. E., Stigum, H., London, S. J., Nystad, W. \& Nafstad, P. (2009). Maternal obesity in pregnancy and respiratory health in early childhood. Paediatric and perinatal epidemiology, 23(4), 352-362. https://doi. org/10.1111/j.1365-3016.2009.01034.x

Hernandez, T. L., Friedman, J. E., Barbour, L. A. (2020) Insulin Resistance in Pregnancy: Implications for Mother and Offspring. En: Zeitler, P., Nadeau, K. (eds) Insulin
Resistance. Contemporary Endocrinology. Humana, Cham. https://doi.org/10.1007/978-3-030-25057-7_5

Isa, T., Yamane, I., Hamai, M. \& Inagaki, H. (2009). Japanese macaques as laboratory animals. Experimental animals, 58(5), 451-457. https://doi.org/10.1538/expanim.58.451

Kaati, G., Bygren, L. O. \& Edvinsson, S. (2002). Cardiovascular and diabetes mortality determined by nutrition during parents' and grandparents' slow growth period. European journal of human genetics, EJHG, 10(11), 682-688. https://doi.org/10.1038/sj.ejhg.5200859

Kirk, S. L., Samuelsson, A. M., Argenton, M., Dhonye, H., Kalamatianos, T., Poston, L., Taylor, P. D. \& Coen, C. W. (2009). Maternal obesity induced by diet in rats permanently influences central processes regulating food intake in offspring. PloS one, 4(6), e5870. https://doi. org/10.1371/journal.pone.0005870

Knight, B., Shields, B. M., Hill, A., Powell, R. J., Wright, D. \& Hattersley, A. T. (2007). The impact of maternal glycemia and obesity on early postnatal growth in a nondiabetic Caucasian population. Diabetes care, 30(4), 777-783. https://doi.org/10.2337/dc06-1849

Li, Y. (2018). Epigenetic Mechanisms Link Maternal Diets and Gut Microbiome to Obesity in the Offspring. Frontiers in genetics, 9, 342. https://doi.org/10.3389/ fgene.2018.00342

Linares Segovia, B., Gutiérrez Tinoco, M., Izquierdo Arrizon, A., Guízar Mendoza, J. M. \& Amador Licona, N. (2012). Long-term consequences for offspring of paternal diabetes and metabolic syndrome. Experimental diabetes research, 2012, 684562. https://doi.org/10.1155/2012/684562

Lindell, N., Carlsson, A., Josefsson, A. \& Samuelsson, U. (2018). Maternal obesity as a risk factor for early childhood type 1 diabetes: a nationwide, prospective, population-based case-control study. Diabetologia, 61(1), 130-137. https://doi.org/10.1007/s00125-017-4481-2

Lucas, A., Morley, R., Cole, T. J., Gore, S. M., Lucas, P. J., Crowle, P., Pearse, R., Boon, A. J. \& Powell, R. (1990). Early diet in preterm babies and developmental status at 18 months. Lancet (London, England), 335(8704), 14771481. https://doi.org/10.1016/0140-6736(90)93026-1

Lumey, L. H., Stein, A. D., Kahn, H. S. \& Romijn, J. A. (2009). Lipid profiles in middle-aged men and women after famine exposure during gestation: the Dutch Hunger Winter Families Study. The American journal of clinical nutrition, 89(6), 1737-1743. https://doi.org/10.3945/ ajcn.2008.27038

Ma, J., Prince, A. L., Bader, D., Hu, M., Ganu, R., Baquero, K., Blundell, P., Alan Harris, R., Frias, A. E., Grove, K. L. \& Aagaard, K. M. (2014). High-fat maternal diet during pregnancy persistently alters the offspring microbiome in a primate model. Nature communications, 5, 3889. https://doi.org/10.1038/ncomms4889

McCurdy, C. E., Bishop, J. M., Williams, S. M., Grayson, B. E., Smith, M. S., Friedman, J. E. \& Grove, K. L. (2009). 
Maternal high-fat diet triggers lipotoxicity in the fetal livers of nonhuman primates. The Journal of clinical investigation, 119(2), 323-335. https://doi.org/10.1172/ JCI32661

Ng, S. F., Lin, R. C., Laybutt, D. R., Barres, R., Owens, J. A. \& Morris, M. J. (2010). Chronic high-fat diet in fathers programs $\beta$-cell dysfunction in female rat offspring. Nature, 467(7318), 963-966. https://doi.org/10.1038/ nature 09491

Nomura, Y., Lambertini, L., Rialdi, A., Lee, M., Mystal, E. Y., Grabie, M., Manaster, I., Huynh, N., Finik, J., Davey, M., Davey, K., Ly, J., Stone, J., Loudon, H., Eglinton, G., Hurd, Y., Newcorn, J. H. \& Chen, J. (2014). Global methylation in the placenta and umbilical cord blood from pregnancies with maternal gestational diabetes, preeclampsia, and obesity. Reproductive sciences (Thousand Oaks, Calif.), 21(1), 131-137. https://doi. org/10.1177/1933719113492206

Organización Mundial de la Salud (2020, abril 1). Obesidad y sobrepeso. https://www.who.int/es/news-room/factsheets/detail/obesity-and-overweight

Saben, J. L., Boudoures, A. L., Asghar, Z., Thompson, A., Drury, A., Zhang, W., Chi, M., Cusumano, A., Scheaffer, S. \& Moley, K. H. (2016). Maternal Metabolic Syndrome Programs Mitochondrial Dysfunction via Germline Changes across Three Generations. Cell reports, 16(1), 1-8. https://doi.org/10.1016/j.celrep.2016.05.065

Samuelsson, A. M., Matthews, P. A., Argenton, M., Christie, M. R., McConnell, J. M., Jansen, E. H., Piersma, A. H., Ozanne, S. E., Twinn, D. F., Remacle, C., Rowlerson, A., Poston, L. \& Taylor, P. D. (2008). Diet-induced obesity in female mice leads to offspring hyperphagia, adiposity, hypertension, and insulin resistance: a novel murine model of developmental programming. Hypertension (Dallas, Tex.: 1979), 51(2), 383-392. https://doi.org/10.1161/ HYPERTENSIONAHA.107.101477

Schulz, L. C. (2010). The Dutch Hunger Winter and the developmental origins of health and disease. Proceedings of the National Academy of Sciences of the United
States of America, 107(39), 16757-16758. https://doi. org/10.1073/pnas.1012911107

Sebire, N. J., Jolly, M., Harris, J. P., Wadsworth, J., Joffe, M., Beard, R. W., Regan, L. \& Robinson, S. (2001). Maternal obesity and pregnancy outcome: a study of 287,213 pregnancies in London. International journal of obesity and related metabolic disorders: journal of the International Association for the Study of Obesity, 25(8), 1175-1182. https://doi.org/10.1038/sj.ijo.0801670

Secretaría de Salud, Instituto Nacional de Salud Pública (INSP) e Instituto Nacional de Estadística y Geografía (INEGI). Encuesta Nacional de Salud y nutrición: Resultados Nacionales 2018. https://ensanut.insp.mx/ encuestas/ensanut2018/doctos/informes/ensanut_2018_ presentacion_resultados.pdf

Slyvka, Y., Zhang, Y. \& Nowak, F. V. (2015). Epigenetic effects of paternal diet on offspring: emphasis on obesity. Endocrine, 48(1), 36-46. https://doi.org/10.1007/s12020014-0328-5

Soderborg, T. K., Clark, S. E., Mulligan, C. E., Janssen, R. C., Babcock, L., Ir, D., Young, B., Krebs, N., Lemas, D. J., Johnson, L. K., Weir, T., Lenz, L. L., Frank, D. N., Hernandez, T. L., Kuhn, K. A., D’Alessandro, A., Barbour, L. A., El Kasmi, K. C. \& Friedman, J. E. (2018). The gut microbiota in infants of obese mothers increases inflammation and susceptibility to NAFLD. Nature communications, 9(1), 4462. https://doi.org/10.1038/ s41467-018-06929-0

Veenendaal, M. V., Painter, R. C., de Rooij, S. R., Bossuyt, P. M., van der Post, J. A., Gluckman, P. D., Hanson, M. A. \& Roseboom, T. J. (2013). Transgenerational effects of prenatal exposure to the 1944-45 Dutch famine. BJOG: an international journal of obstetrics and gynaecology, 120(5), 548-553. https://doi.org/10.1111/14710528.12136

Zhou, L. \& Xiao, X. (2018). The role of gut microbiota in the effects of maternal obesity during pregnancy on offspring metabolism. Bioscience reports, 38(2), BSR20171234. https://doi.org/10.1042/BSR20171234 